\title{
Value of Ankle-jerk Timing in the Assessment of Thyroid Function
}

\author{
ABRAHAM S. ABRAHAM,* M.B., M.R.C.P. ; MICHAEL ATKINSON, $\dagger$ M.D., M.R.C.P. \\ BRUCE ROSCOE, $\ddagger$ M.B., CH.B.
}

Brit. med. F., 1966, 1, 830-833

The delayed relaxation of the tendon-jerk in myxoedema was first described by Ord (1884), and in 1924 Chaney, using a pneumatic recording system, measured the tendon-reflex time in myxoedema and showed that it returned to normal with treatment. In 1929 Mussio Fournier demonstrated rapid tendon reflexes in thyrotoxicosis.

Various techniques have since been devised for timing the ankle-jerk. The movement of the foot has been transmitted to a pedal recording electronically (Lambert et al., 1951 ; Smart and Robson, 1963 ; Miles and Surveyor, 1965), or to a wire moving through an electrolyte solution which completes an electrical circuit (Moulopoulos et al., 1964). The photoelectric method (Photomotograph) of Gilson (1959), depending upon interruption by the foot of a beam of light activating a photoelectric cell, has been used by Fogel et al. (1962), Avera and Overholt (1962), Sherman et al., (1963), and Simpson et al. (1963). The electromagnetic system (Kinemometer) of Lawson (1958), in which movement of a magnet attached to the foot induces a current in an adjacent coil, was used by de Gennes and Deschamps (1961), Mann (1963), and Nuttall and Doe (1964). This apparatus commended itself to us because of its simplicity and convenience, and has been employed in this present study.

It is now generally established that the tendon reflexes are shortened in the majority of thyrotoxic patients and prolonged in untreated myxoedema. The present survey was made to assess the value of tendon-reflex measurements in patients with thyroid dysfunction, with particular reference to the differential diagnosis of anxiety from thyrotoxicosis and to their value in regulation of treatment and in the assessment of thyroid function in pregnancy, when the protein-bound iodine (P.B.I.) is often raised.

\section{Material}

A control group consisting of 129 males and 131 females, made up of hospital staff, students, and ambulant patients recovering from minor medical and surgical conditions, was studied.

Thirteen patients suffering from myxoedema, and 30 patients who were eventually proved to have thyrotoxicosis, were investigated, together with euthyroid patients originally referred because of suspected thyrotoxicosis (36) or myxoedema (21). The Wayne (1960) index was calculated and serum proteinbound iodine estimations were made in nearly all patients; radioiodine studies were used only when doubt remained about the diagnosis.

Serial ankle-jerk times were recorded during treatment of myxoedema with thyroxine in four patients, and during treatment of thyrotoxicosis with carbimazole (three), radioiodine and carbimazole (four), or with Lugol's iodine prior to thyroidectomy (two).

Tendon-jerks were timed in the antenatal clinic in 140 women at various stages of pregnancy, and P.B.I. estimations were done in 33 taken at random.

* Medical Registrar, Ronkswood Hospital, Worcester. f Consultant Physician, Ronkswood Hospital, Worcester.
Measurements were made on 83 diabetics attending the outpatient clinic.

\section{Method}

The method used was that of Lawson (1958), in which the ankle-jerk is timed by means of a magnet attached to the sole, movement of which generates current in an adjacent coil recording directly on an electrocardiograph.

With the subject kneeling on a chair, a horseshoe magnet was strapped to the sole of the foot under the arch. At the level of its poles, and $2 \mathrm{~cm}$. distant, were placed twin induction coils wound on soft iron cores connected in series and attached direct to two leads of a portable electrocardiograph (Fig. 1). Though the magnitude of the deflection recorded on the electrocardiograph when the ankle-jerk was elicited varied with the distance of the coil from the magnet, the sensitivity of the coil, the force of the blow, and the amplitude of the tendon-jerk, its time relationships remained constant.

The paper speed of the electrocardiograph was always verified, and, though more accurate timing was possible when this could be increased to $50 \mathrm{~mm} . / \mathrm{sec}$., the practical advantage thus obtained was slight, and we used mainly a portable machine running at $25 \mathrm{~mm} . / \mathrm{sec}$.

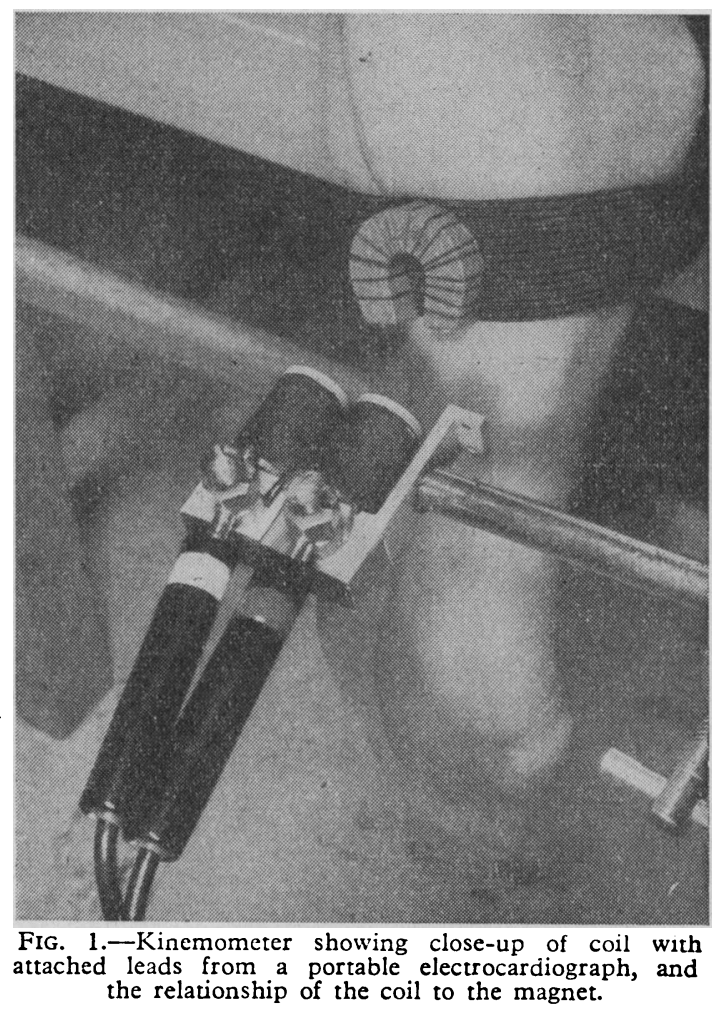

\section{Measurements}

The tracing obtained (Fig. 2) consists of initial rapid oscillations due to the hammer striking the tendon, beginning 
at point $S$, followed by a slower downward deflection caused by muscle contraction, and a final upward deflection representing relaxation. The terminology of Nuttall and Doe (1964) was used, the valley being designated $\mathrm{V}$ and the peak $\mathrm{P}$; both these points are easily identifiable.

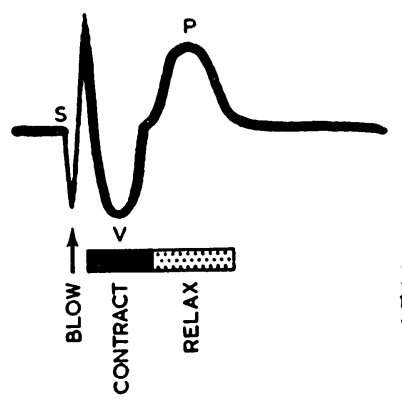

FIG. 2.-Diagrammatic representation of the ankle-ierk, showing the contraction and relaxation phases.

Initially the distances SP, SD (D being the point where the line VP crosses the isoelectric line), SE (E being the end of the relaxation phase), and VP were measured. However, we found that $\mathrm{D}$ was variable and $\mathrm{E}$ often difficult to identify. The interval VP was the best in discriminating patients with thyroid disease from the control group, which is in accordance with the findings of Nuttall and Doe (1964). The VP interval is that between maximal rate of contraction and maximal rate of relaxation, and, unlike the other values (SP, SE, and SD), or measurements by other methods, is an index solely of the muscle response, being quite independent of nerve conduction rate.

A notch was commonly seen on the VP upstroke, but its presence did not prolong the VP interval. However, if the coil was placed much above or below the level of the poles of the magnet the notch became so large as to cause difficulty in identifying $\mathrm{V}$ and $\mathrm{P}$ and could lead to falsely low readings of the VP interval.

\section{Results}

Control Group (Table I).-The mean value for VP in the control group of subjects was 193 msec., and the normal range was taken as $120-300$ msec. No significant variation occurred with sex or age. In the individual the VP interval never varied by more than $20 \mathrm{msec}$. between the left and right ankles, and serial measurements over periods of several weeks showed comparably small variation.

Effect of Ankle Oedema.-Ankle-reflex times were recorded in three patients with congestive cardiac failure and gross ankle oedema. In each, treatment with diuretics caused the ankle-

\begin{tabular}{|c|c|c|c|c|}
\hline & & & No. & Mean VP (msec.) \\
\hline $\begin{array}{l}\text { Males } \\
\text { Females }\end{array}$ & $\therefore$ & $\begin{array}{ll} & . \\
. & \end{array}$ & $\begin{array}{l}129 \\
131\end{array}$ & $\begin{array}{l}189 \pm 40 \\
197 \pm 37 \cdot 1\end{array}$ \\
\hline . & Total & $\ldots$ & 260 & $193 \pm 35 \cdot 1$ \\
\hline
\end{tabular}

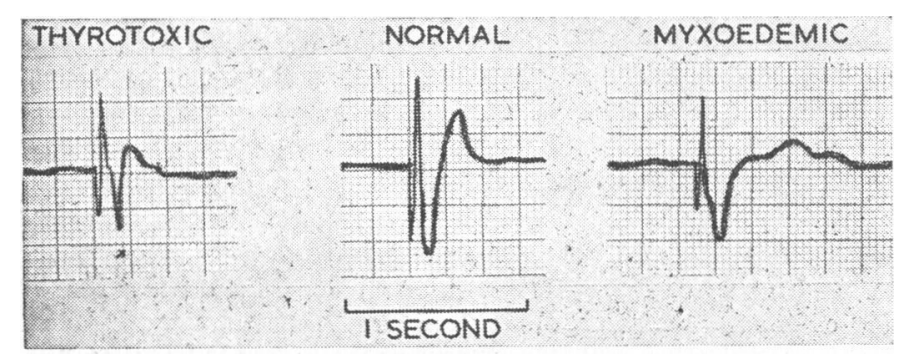

FIG. 3.-Specimen recordings from normal (VP $160 \mathrm{msec}$ ), thyrotoxic (VP $60 \mathrm{msec}$ ), and myxoedematous (VP $400 \mathrm{msec}$.) patients. jerk to quicken as oedema disappeared. In the first two patients the VP time fell from 320 and $300 \mathrm{msec}$. to 260 and $240 \mathrm{msec}$. respectively; the third patient suffered from thyrotoxicosis and congestive cardiac failure (see below). Ankle-reflex times were compared in the two legs of a patient with unilateral oedema due to venous thrombosis. The VP value in the oedematous leg was $240 \mathrm{msec}$. compared with $160 \mathrm{msec}$. in the unaffected leg. Following treatment, and after loss of oedema, the VP interval on both sides was $160 \mathrm{msec}$. Hence gross ankle oedema causes a prolongation of ankle-jerk time and must be taken into account in interpretation of results.

Suspected Thyroid Dysfunction.-Typical ankle-jerk recordings in thyrotoxicosis and myxoedema are shown in Fig. 3. The SV time, which depends chiefly upon nerve-conduction time, was not abnormal in either thyrotoxicosis or myxoedema.

Myxoedema.-In myxoedema the final upward deflection caused by relaxation was prolonged. The phase of contraction was neither delayed nor greatly prolonged, hence the $S V$ and SD measurements were normal. The VP interval was prolonged in each of the 13 patients with proved myxoedema (Fig. 4), the mean value for this group being $418 \mathrm{msec}$. and the shortest VP $320 \mathrm{msec}$. At the time of measurement two had slight ankle oedema. Ankle-jerk prolongation, therefore, was a constant finding in myxoedema. In a further 21 patients the diagnosis of hypothyroidism was considered but eventually
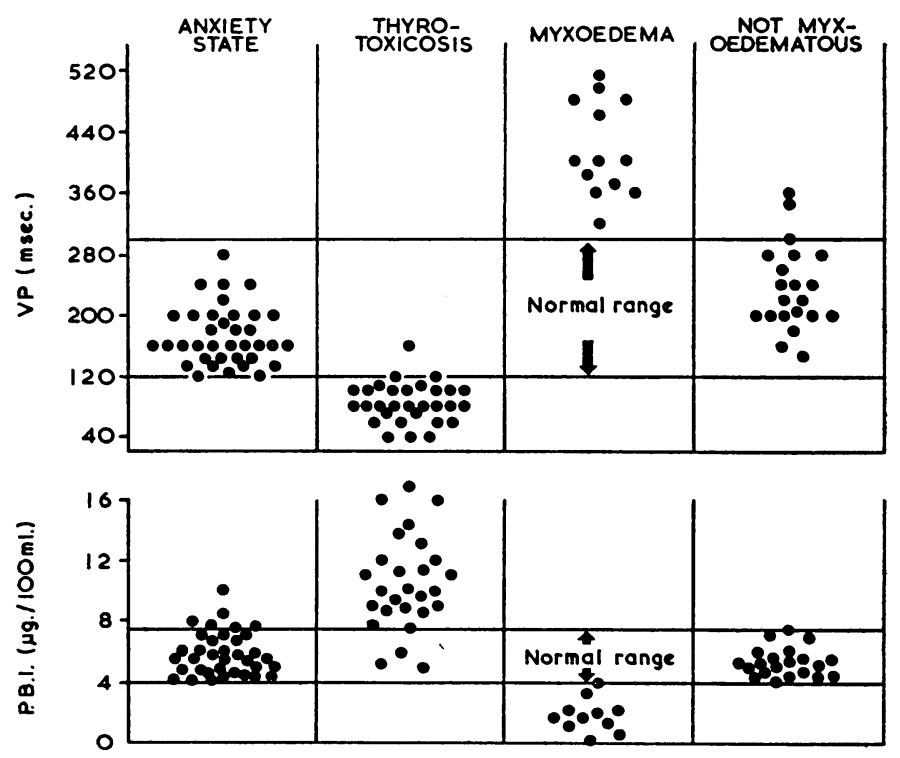

FIg. 4.-VP and P.B.I. values in 100 patients with suspected thyroid dysfunction.

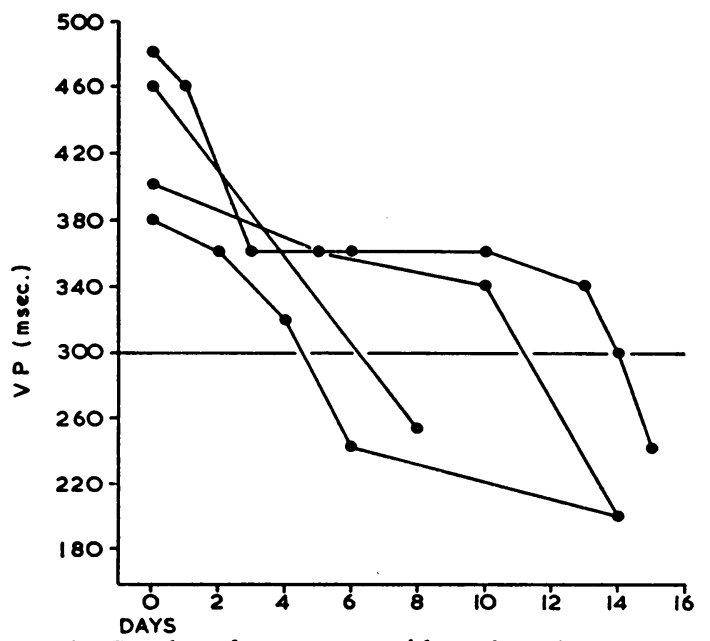

FIG. 5.-Results of treatment with L-thyroxine in four patients with myxoedema, showing the rate of return of the VP times to normal values. 
rejected, and 19 of these had normal VP times, while in two the VP times were outside the normal range at 340 and 360 msec. respectively. Both these patients had cerebral arteriosclerosis with generalized mental slowing and Parkinsonian features, but no oedema. The effects of L-thyroxine were followed, and Fig. 5 illustrates the results in four cases. They are not strictly comparable, as different doses of L-thyroxine were used in each case, but the VP time in all four cases returned to normal within a fortnight of initiating treatment.

Thyrotoxicosis.-In 27 of the 30 patients later proved to have thyrotoxicosis the ankle-jerk time was subnormal, and the VP varied in them between 40 and $110 \mathrm{msec}$., while in two others borderline low values of $120 \mathrm{msec}$. were obtained (Fig. 4). The remaining patient had a VP interval of $160 \mathrm{msec}$., and was in cardiac failure with gross ankle oedema, as was one of the two patients who originally had a VP time of $120 \mathrm{msec}$., in whom serial measurements were made during treatment. Digoxin and bendrofluazide produced a diuresis with initial quickening of the tendon-jerk (VP=100 $\mathrm{msec}$.) before this became prolonged again as a result of antithyroid treatment. Serial anklejerk times were measured in these patients after thyroidectomy, and during treatment with carbimazole, alone or combined with radioiodine (Fig. 6). The tendon-jerk times returned to normal ahead of the patients' clinical state and were an early indication of satisfactory response.

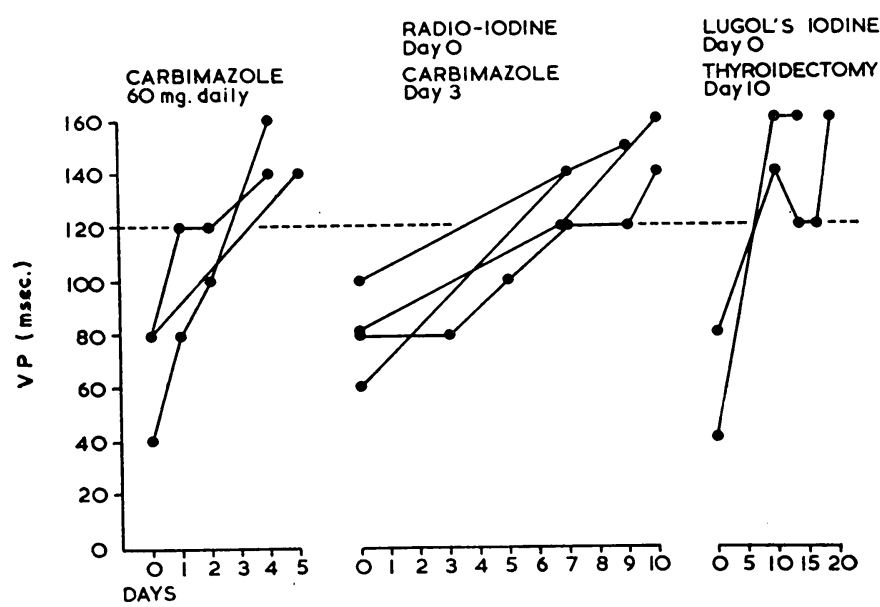

PIG. 6.- Treatment of thyrotoxicosis with carbimazole alone, radioiodine and carbimazole, and Lugol's iodine prior to thyroidectomy, showing the rate at which VP times returned to normal in each case.

Anxiety States (Fig. 4).--In 36 patients the final diagnosis was anxiety state, though in each the possibility of thyrotoxicosis had been raised. In none was the ankle-jerk time outside the normal range.

Comparison of the Diagnostic Accuracy of Ankle-jerk Time and Serum P.B.I. (Fig. 4).-The ankle-jerk time was not quite as accurate as the P.B.I. in the diagnosis of myxoedema, since prolonged VP times were recorded in two non-myxoedematous patients who suffered from advanced, generalized arteriosclerosis, but who had no oedema. In the diagnosis of thyrotoxicosis the ankle-jerk method compared favourably with the P.B.I. ; the latter was normal in three patients with thyrotoxicosis, one of whom had been treated with prednisone for myopathy later proved thyrotoxic, and raised in six patients with anxiety symptoms because of previous medication with iodinecontaining compounds or coexistent liver disease. On the other hand, the VP time was within the normal range in only one thyrotoxic patient, and he was oedematous at the time.

Pregnancy.-One hundred and forty women in all stages of pregnancy were seen and none had thyroid dysfunction. The mean VP was $197.4 \mathrm{msec}$, and there was no statistical difference between this group and normal non-pregnant females under the age of 40 . The mean VP did not differ in the first, second, and third trimesters (Table II). The P.B.I. was estimated in 33 women chosen at random, and it will be seen from Fig. 7 that, though this was often raised, virtually all the tendon-jerk times were within the normal range.

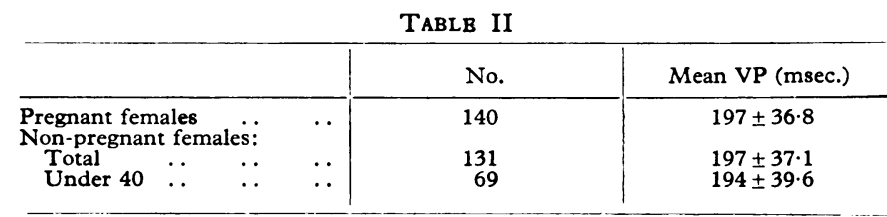

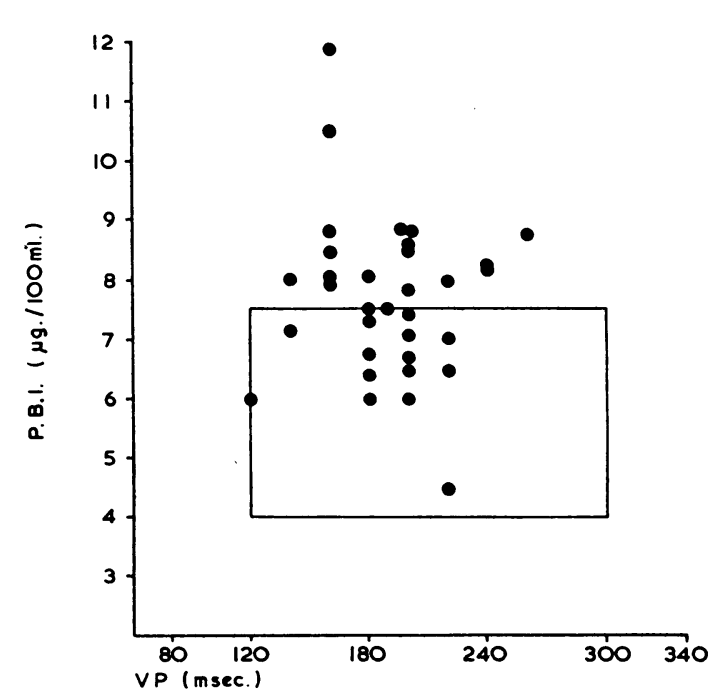

FIG. 7.-Correlation between P.B.I. and VP values in 33 women, chosen at random, during all stages of pregnancy. The square encloses the normal range.

Diabetes.-A total of 83 diabetics of various ages were seen consecutively, both in the wards and in the out-patient clinics. No jerk could be obtained in either ankle in 27 of these patients. In the remaining 56 the VP times were all within our normal range, though the mean VP of $214 \mathrm{msec}$. was slightly higher than the mean in the control group. Presence or absence of the tendon jerk and the VP time did not appear to depend upon the age, sex, duration of diabetes, ease of control, or type of treatment.

\section{Discussion}

There is a great need for a simple test of thyroid function which can be used in domiciliary practice and in peripheral hospitals where other methods are not readily available. Measurement of serum P.B.I. is probably the most widely employed test of the thyroid function, particularly in areas remote from radioisotope centres. Neither radioiodine studies nor P.B.I. estimations measure the end-organ response to thyroid hormone, and the diagnostic value of both may be vitiated by the previous administration of iodine-containing compounds even several years previously (Brown and Westermeyer, 1955) or by non-thyroid disease. Thus a raised P.B.I. has been reported in liver disease (Kydd and Man, 1951 ; Vannotti and Béraud, 1959). Low values are found during salicylate or mercurial therapy (Meyers and Man, 1951), in the nephrotic syndrome where the binding protein in the blood is deficient (Recant, 1956), and during the administration of corticosteroids (Hill et al., 1950). Serum cholesterol and basal metabolic rate are measures of end-organ response, but are of limited value in the diagnosis of thyroid dysfunction.

Tendon-reflex timing is another method of measuring endorgan response, and has been used increasingly in recent years as an index of thyroid function. It has the advantages of giving an immediate result, and, being largely independent of iodine metabolism, it is unaffected by iodine administration except where this suppresses the output of thyroid hormone. 
In general, reflex times correlate well with B.M.R. estimations (Lambert et al., 1951), P.B.I. levels (Sherman et al., 1963), and radioiodine uptake (Sherman et al., 1963 ; Miles and Surveyor, 1965), and are affected little by alteration in metabolic rate (Fogel et al., 1962), or muscle temperature.

The several different methods used in recording tendon-jerks to assess thyroid function have given rise to some conflict of opinion about the value of this test, and Simpson et al. (1963), who found poor reproducibility of times obtained, listed the difficulties encountered with photomotography. The method used in this study measures only the duration of a part of the muscle response in the ankle-jerk-that is, the interval between maximal rate of contraction and maximal rate of relaxation. Most of the other methods employed have measured from the blow on the tendon to the point of half-relaxation, and so include the latent period. Since nerve-conduction is normal in the majority of patients with thyroid dysfunction, its inclusion in the measured time does not increase diagnostic accuracy and may partially hide changes in duration of the muscle response. This is the probable explanation of the finding of Nuttall and Doe (1964), confirmed by our own observations, that in the diagnosis of thyroid dysfunction the VP interval is greatly superior to the SD or SP measurements (both of which include the latent period). Furthermore, nerveconduction may be affected by non-thyroid disease, and this may be the explanation of the delayed Achilles reflex reported in diabetes by Beardwood and Schumacher (1964), who measured the time from the blow on the tendon to halfrelaxation. Mulder et al. (1961) have demonstrated delayed nerve-conduction in diabetes even in the absence of clinical evidence of neuropathy. In our experience the VP interval (which does not include the nerve-conduction time) is not prolonged in diabetes.

The surprising accuracy of ankle-jerk timing in the diagnosis of thyrotoxicosis reported by Nuttall and Doe (1964) has been fully confirmed by our own findings. The final diagnosis of whether thyrotoxicosis was present was made by the clinician in charge, quite independently of this investigation and usually in ignorance of the ankle-jerk time value. In all doubtful cases the thyroid uptake of ${ }^{131} \mathrm{I}$ and the 48-hour protein-bound ${ }^{131}$ I were used for the final diagnosis.

Apart from the care necessary to identify the points $V$ and $P$ in the ankle-jerk tracing, the greatest difficulty is encountered in the oedematous patient. Harrell and Daniel (1941) reported doubling of tendon-reflex relaxation time in an oedematous arm compared with the normal arm in a patient with unilateral oedema without thyroid disease. Our observations in cardiac failure and unilateral deep-vein thrombosis in the leg bear out their findings and emphasize that caution must be used in the interpretation of tendon-jerk times in the grossly oedematous limb. This increase in tendon-reflex time presumably results from the splinting effect of the oedema fluid; it does not occur with mild oedema, and, if possible, measurements should be made after drainage of oedema by elevation of the limb or pressure bandage.

Though previous authors (Lambert et al., 1951 ; Fogel et al., 1962) have reported that return of the reflex speed to normal during treatment of hyperthyroidism or hypothyroidism correlates well with the clinical state of the patient, we have not been able to confirm this. Indeed, in our series, in both thyrotoxic and myxoedematous patients, the VP measurements returned to normal values within a few days of starting treatment and long before any subjective or objective clinical improvement. Furthermore, during treatment of myxoedema or thyrotoxicosis the tendon-jerk time became abnormal some days before clinical evidence of overdosage or underdosage appeared. Hence it is of great value in the regulation of treatment.

The diagnosis of thyroid dysfunction in pregnancy can be difficult to substantiate in the laboratory. In normal pregnancy increased levels of P.B.I. are found as early as the third to sixth week of gestation (Freedberg et al., 1957), and radioiodine uptake increases early in gestation (Pochin, 1952). On the one hand Engstrom and Markardt (1954) have suggested that the increased elaboration of oestrogen may be an important factor in the elevated concentration of P.B.I., while, on the other, Lawson (1958) reported shortening of the contraction phase of the tendon-jerk after administration of high doses of oestrogen. However, though most of the patients seen had raised P.B.I. levels, their tendon-jerk times were all within the normal range. Thus measurement of the reflex time appears to be a reliable test of thyroid function in pregnancy, and certainly a safe one.

\section{Conclusion}

A simple, cheap, and entirely portable method is described for the measurement of the tendon-reflex time. It is shown to be a reliable diagnostic aid in thyroid dysfunction and particularly in cases where clinical difficulty is present.

Attention is drawn to the possibility of error in patients with ankle oedema, which tends to increase the VP interval significantly.

The measurement of the tendon-jerk time is a reliable test of thyroid function in pregnancy.

ADDENDUM.-Since submitting this paper for publication the total number of patients with proved thyrotoxicosis we have studied has risen to 44 , and in 38 of these the VP time was $120 \mathrm{msec}$. or less. In three patients ankle oedema was present and the initial VP times were 160,160 , and $140 \mathrm{msec}$. respectively, falling to subnormal levels after disappearance of the oedema. In the remaining three patients tendon-jerk values within the normal range were obtained in the absence of oedema, and each rose further with antithyroid treatment. We have now studied 22 patients with myxoedema, and in each the VP time has been $300 \mathrm{msec}$. or more.

We should like to thank the many clinicians who have allowed us to study patients under their care; Dr. F. J. Pick and Mr. H. Michell, of Staffordshire General Infirmary, for the P.B.I. estimations; and Dr. G. A. Newsholme, of the Queen Elizabeth Hospital, Birmingham, for the radioiodine studies. We are indebted to $\mathrm{Mr}$. G. D. Holland for the photographs.

\section{REFERENCES}

Avera, J. W., and Overholt, B. M. (1962). Amer. Practit., 13, 251. Beardwood, D. M., and Schumacher, L. R. (1964). Amer. f. med. Sci. $247,324$. Brown, H. B., and Westermeyer, V. W. (1955). Cleveland Clin. Quart.,

Chaney, W. C. (1924). F. Amer. med. Ass., 82, 2013.

de Gennes, L., and Deschamps, H. (1961). Presse méd., 69, 1543.

Engstrom, W. W., and Markardt, B. (1954). F. clin. Endocr., 14, 215.

Fogel, R. L., Epstein, J. A., Stopak, J. H., and Kupperman, H. S. (1962). N.Y. St. F. Med., 62, 1159.

reedberg, I. M., Hamolsky, M. W., and Freedberg, A. S. (1957). New Gilson. W. E. (1959)

Gilson, W. E. (1959). Ibid., 260, 1027.

Harrell, G. T., and Daniel, D. (1941). N.C. med. F., 2, 549

Hill, S. R., iun, Reiss, R. S., Forsham, P. H., and Thorn, G. W. (1950). f. clin. Endocr., 10, 1375.

Kydd, D. M., and M̈an, E. B. (1951). F. clin. Invest., 30, 874.

Lambert, E. H., Underdahl, L. O., Beckett, S., and Mederos, L. 0. (1951). F. clin. Endocr., 11, 1186.

Lawson, J. D. (1958) New Engl. 7. Med., 259, 761.

Mann, A. S. (1963). Amer. f. med. Sci., 245, 317

Meyers, J. H., and Man. E. B. (1951). F. Lab. clin. Med., 37, 867

Miles. D. W., and Surveyor, I. (1965). Brit. med. f., 1, 158. Moulopoulos, S. D., Koutras, D. A., and Kralios, A. C. (1964). Lancet, 1,85

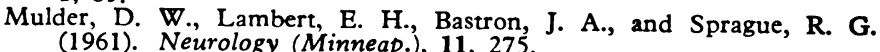
Mussions . Neurology (Minneap.), 11, 275

Mussio Fournier, J. C. (1929). Quoted by Nuttall and Doe (1964).

Nuttall, F. Q., and Doe, R. P. (1964). Ann. intern. Med., 61, 269.

Prd. W. M. (1884). Brit. med. f., 2, 205.

Pochin, E. E. (1952). Clin. Sci., 11, 441.

Recant, L. (1956). 7. clin. Invest., 35, 730.

Sherman, L., Goldherg, M., and Larson, F. C. (1963). Lancet, 1, 243. pson, G. M., Blair, J. H., and Nartowicz, G. R. (1963). N.Y.'St. Y. Med., 63, 1148 .

Smart, G. A., and Robson, A. M. (1963). Lancet, 1, 363.

Wannotti, A., and Béraud, T. (1959). ₹. clin. Endocr., 19, 466. 